\title{
IgA dominant glomerulonephritis associated to staphylococcus infection: a peculiar case report
}

\author{
Nicole Pestana ${ }^{1}$, Francisca Silva ${ }^{1}$, Pedro Vieira ${ }^{1}$, Ricardo Figueira ${ }^{2}$, José Durães ${ }^{1}$, Gil Silva ${ }^{1}$ \\ ${ }^{1}$ Department of Nephrology of Hospital Central do Funchal, Funchal, Portugal \\ 2 Department of Rheumatology of Hospital Central do Funchal, Funchal, Portugal \\ Avenida Luís de Camões no 57, 9004-514, Funchal
}

\section{ABSTRACT}

IgA dominant glomerulonephritis associated to Staphylococcus infection is a rare clinical entity that has been described mainly in case reports. Biopsy features can resemble other disease entities mainly IgA nephropathy and Henoch-Schönlein purpura nephritis. Treatment of IgA dominant glomerulonephritis associated to staphylococcal infection is based on antibiotics for the underlying infection, controlling hypertension and edema and may resort to concomitant use of steroids in selected cases. Prognosis markers such as hypertension, diabetes and interstitial fibrosis may influence treatment as they are associated with poor renal outcomes. We report a case of a 63-year-old man with known hypertension, pre-diabetes and recent history of methicillin-sensitive Staphylococcus aureus bacteremia associated to prostatitis, who presented with a one-month history of edema, arthralgia and foamy urine. Over this period he progressed to anasarca and nephrotic range proteinuria with concomitant rise in creatinine levels being documented. The renal biopsy showed segmental endocapillary proliferation and IgA segmental dominant staining associated to C3 and lambda in minor distribution. On completion of two months of steroid therapy the patient partially recovered his renal function and proteinuria. After nine months of tapering steroids, he presented with acute inflammatory arthritis supporting an inflammatory background disease. To our knowledge this case describes an unusual entity such as IgA dominant glomerulonephritis associated to staphylococcal infection co-presenting with an associated reactive arthritis.

Keywords: IgA nephropathy, Staphylococcus, Postinfectious glomerulonephritis

\section{INTRODUCTION}

IgA dominant glomerulonephritis (GN) associated to staphylococcal infection while rare is an increasingly recognized morphologic variant of infectious $\mathrm{GN}$, in which there is glomerular deposition of IgA, either dominant or co-dominant. This entity has been described mainly through minor case reports. ${ }^{1}$ Koyama et al first described it in 1995, in 10 patients with methicillin-resistant Staphylococcus aureus (MRSA) infection. ${ }^{2}$ Methicillin-sensitive Staphylococcus aureus (MSSA) has also been reported in $18 \%$ of patients. ${ }^{2}$

It is thought that bacterial superantigens, such as enterotoxins ( $C$ and $A$ ) and toxic shock syndrome toxin act as activators of the immune system, leading to the formation of immunocomplexes. ${ }^{3}$ By binding to major histocompatibility class II molecules they stimulate T cells and cytocine production, inducing polyclonal activation of IgG and $\operatorname{lgA} .^{2}$ Koyama et al also suggested that a probable adhesion factor, defined as Staphylococcus aureus cell envelope antigen, has a pathogenic induction role since it has been noticed in patients with $\lg A$ nephropathy (IgAN) and IgA dominant GN associated to staphylococcal infection but not in other immunocomplex glomerulonephritis. ${ }^{4}$

IgA dominant GN associated to staphylococcal infection is most frequently seen in elderly male patients and over half are diabetic. ${ }^{5}$ Staphylococcus has become 3 times more common than Streptococcus as the causative agent of infectious glomerulonephritis in the elderly. 6

The origin of infection is highly variable, with the most frequent skin, deep-seated abscesses, surgical wounds, lung, joint and heart. ${ }^{1,5}$ Features backing IgA dominant $\mathrm{GN}$ associated to staphylococcal infection include initial presentation at older age, diabetes, acute renal failure, intercurrent culture documenting staphylococcal infection, hypocomplementemia, diffuse glomerular endocapillary hypercellularity on light microscopy (LM), dominant immunofluorescent (IF) staining for C3 over IgA and presence of subepithelial humps on electron microscopy (EM). 7,8 Immunocomplex mediated glomerulonephritis and IgAN have been described as rare entities associated to seronegative spondyloarthropathies. Concurrently reactive arthritis and glomerulonephritis are rare among the adult population and often underdiagnosed. Different infectious agents can trigger reactive arthritis, usually affecting people in their second to fourth decades of life and occurs weeks following genitourinary (male to female 9:1) or enteric (1:1) infections. ${ }^{9}$ Most of these patients respond to non-steroid anti-inflammatory drugs while steroids are effective in peripheral arthritis and aggressive presentation cases.

Regarding the emerging importance of a new entity such as IgA dominant GN associated to Staphylococcus aureus infection we present a patient who had an overlooked MSSA infection history with rapidly 
progressive renal dysfunction associated to a classical histological pattern of IgA dominance.

\section{CASE REPORT}

A 63-year-old caucasian man was referred to our emergency department due to routine laboratory finding of a stage II acute kidney injury $(\mathrm{AKI})^{10}$. His medical background was marked by a history of hypertension, diagnosis of pre-diabetes 8 years ago and a recent hospital admission dating back 3 months with a MSSA bacteremia due to prostatitis. At that time, he presented normal kidney function and no proteinuria. His history was otherwise unremarkable as he had no relevant family history and was currently on no chronic medication.

One month before emergency admission the patient referred a prior eight-kilogram weight gain and starting of progressive peripheral edemas, foamy urine and polyarticular pain in small and medium joints with an inflammatory pattern. Apart from the edema, his physical examination was unremarkable, namely for afebrile and normotensive presentations and no skin rash was noticed. Laboratory findings had already shown altered renal function with serum creatinine $(\mathrm{sCr})$ of $1.8 \mathrm{mg} / \mathrm{dL}$, hypercholesterolemia and hypertriglyceridemia. Urinalysis was normal, with no proteinuria or hematuria. A laboratory re-evaluation was requested within a one-month period that evidenced an abrupt rise in $\mathrm{s} C r$ motivating emergency department referral.

On admission to the emergency department, laboratory tests evidenced a rapidly progressive renal failure, with $\mathrm{sCr}$ of $3.62 \mathrm{mg} / \mathrm{dL}$ as opposed to a 3-months earlier basal level of $0.8 \mathrm{mg} / \mathrm{dL}$ and $1.8 \mathrm{mg} / \mathrm{dL}$ one month before. As for urinalysis, an active sediment was now evident showing $3+$ erythrocyturia with a nephrotic range proteinuria $(4+$ with a urinary protein/creatinine ratio of $23 \mathrm{mg} / \mathrm{mg}$ ) and a serum

\section{Figure 1}

Glomerulus showing mesangial and endocapillary proliferation and infiltrating leucocytes. Periodic acid-Schiff, $\times 400$

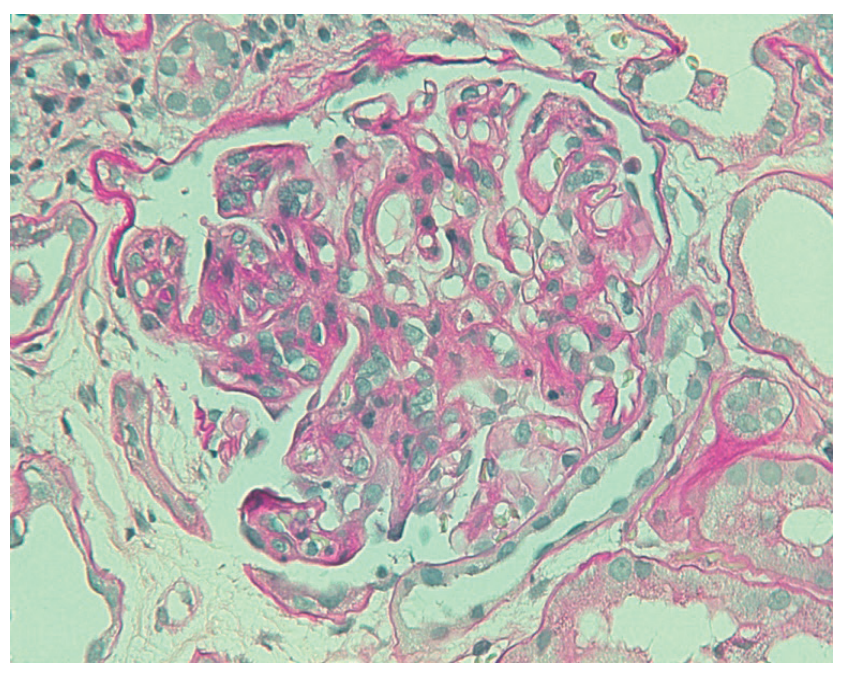

albumin of $22 \mathrm{mg} / \mathrm{dL}$. Subsequent investigation showed no abnormal diagnostic imaging findings on renal ultrasound and a negative serology for viral hepatitis, HIV and VDRL. Immunological markers (antinuclear antibody (ANA) (IIF (Indirect Immunofluorescence) and CLIA (chemiluminescent immunoassay), anti-double-stranded DNA (anti-dsDNA) (IFI and CLIA), antineutrophil cytoplasmic antibody (ANCA) (CLIA) were normal. No complement changes were detected and his serum protein electrophoresis showed low albumin, elevated alpha 2 and beta region and decreased gamma region, resembling a nephrotic pattern. Cryoglobulinemia was not screened. Diabetic retinopathy was excluded to assess microvascular diabetic involvement. At this time salt restriction, inhibitor enzyme angiotensin-converting, loop diuretics, statin and low molecular weight heparin (LMWH) were initiated followed by a kidney biopsy on day 3 (with temporary suspension of $\mathrm{LMWH}$ ) due to a clinical course of rapidly progressive glomerulonephritis. The biopsy showed segmental endocapillary proliferation (Figure 1) and fibrinoid necrosis. Extensive tubular necrosis was noted. IF revealed dominant IgA segmental deposition (Figure 2), associated to C3 and lambda in minor distribution. Kappa light chain was absent. EM was not performed. This histopathological finding associated to his clinical background of diabetes, documented prior 10-week infection of MSSA bacteremia and an aggressive presentation led to the suspicion of IgAdominantGN associated to staphylococcal infection.

Due to his highly active inflammatory histopathological background and no signs of active infection, we opted for a trial of $1 \mathrm{mg} / \mathrm{kg} /$ day prednisone $(60 \mathrm{mg})$ for 8 weeks, then tapered off over a 9-month period. Gradual improvement of his renal function was documented, and after 52 weeks his sCr was $1.8 \mathrm{mg} / \mathrm{dL}$ (maximum sCr level $4.84 \mathrm{mg}$ / $\mathrm{dL}$ ) (Table I). Though, following steroids withdrawal and apparent renal recovery with no signs of clinical activity (lower level of hematuria and total remission of proteinuria and normal complementemia), the arthralgia relapsed following the same previous pattern but with a

\section{Figure 2}

Anti-lgA immunofluorescence showing granular glomerular capillary wall and mesangial staining for $\lg A$

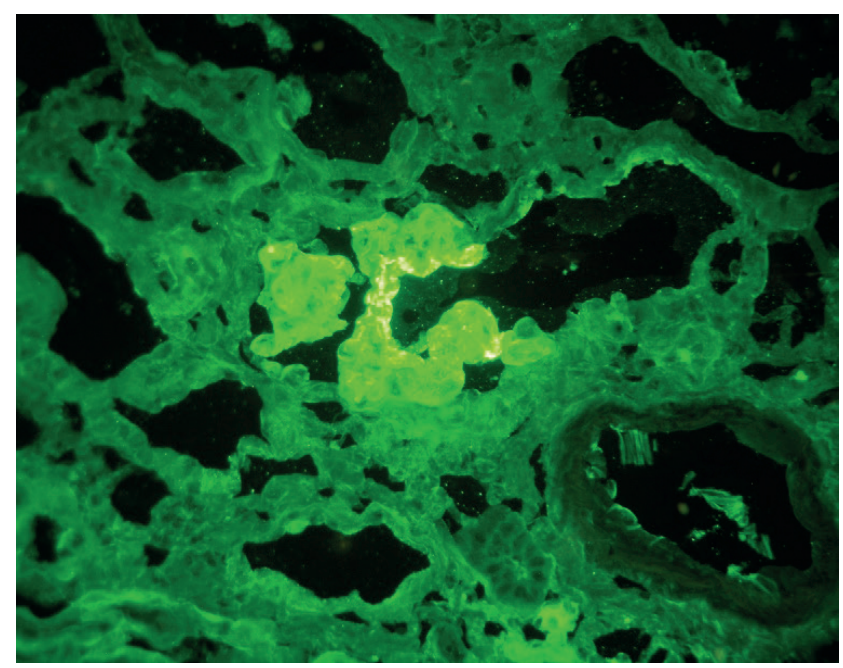


Table I

Laboratorial data showing patient's evolution

\begin{tabular}{|c|c|c|c|c|c|c|c|c|c|c|}
\hline Variable & $\begin{array}{l}\text { Reference } \\
\text { range }\end{array}$ & $\begin{array}{l}1 \text { month } \\
\text { before } \\
\text { hospital } \\
\text { admission }\end{array}$ & $\begin{array}{l}\text { On hospital } \\
\text { admission }\end{array}$ & $\begin{array}{c}\text { Following } \\
1 \text { week } \\
\text { investigation }\end{array}$ & $\begin{array}{c}\text { Day } 5 \\
\text { Prednisolone } \\
\text { (60mg) }\end{array}$ & $\begin{array}{c}\text { Day } 10 \\
\text { Prednisolone } \\
(60 \mathrm{mg})\end{array}$ & $\begin{array}{c}2 \text { months } \\
\text { Prednisolone } \\
\text { (50mg) }\end{array}$ & $\begin{array}{c}4 \text { months } \\
\text { Prednisolone } \\
\text { (30mg) }\end{array}$ & $\begin{array}{c}9 \text { months } \\
\text { Prednisolone } \\
(5 \mathrm{mg})\end{array}$ & $\begin{array}{l}12 \text { months } \\
\text { Prednisolone } \\
\text { (0mg) }\end{array}$ \\
\hline \multicolumn{11}{|c|}{ Blood } \\
\hline Hemoglobin (g/dl) & $13.0-18.0$ & 13.4 & 13.9 & 12.2 & 12.4 & 12.4 & 9.4 & 9.2 & 10.4 & 10.2 \\
\hline White-cell count (per mm³) & $4500-11,000$ & 7,700 & 9,900 & 7,900 & 14,600 & 24,300 & 15,700 & 11,800 & 10,100 & 12,300 \\
\hline Platelet count (per mm³) & $\begin{array}{c}150,000- \\
450,000\end{array}$ & 269,000 & 239,000 & 302,000 & 291,000 & 249,000 & 178,000 & 184,000 & 232,000 & 272,000 \\
\hline $\begin{array}{l}\text { Erythrocyte-sedimentation } \\
\text { rate }(\mathrm{mm} / \mathrm{hr})\end{array}$ & $0-20$ & - & - & 80 & 59 & 52 & 54 & 35 & 69 & 74 \\
\hline C-reactive protein (mg/dL) & $<6.10$ & - & $0-21$ & 2.76 & 0.61 & 0.69 & 0.44 & 0.40 & 6.66 & 16.91 \\
\hline Urea nitrogen (mg/dl) & $8.0-50.0$ & 45 & 73 & 55 & 207 & 137 & 139.9 & 174.7 & 150.2 & 129.0 \\
\hline Creatinine (mg/dL) & $0.7-1.20$ & 1.8 & 3.62 & 3.4 & 4.84 & 2.96 & 1.87 & 2.07 & 2.02 & 1.86 \\
\hline Total protein (g/dl) & $60.0-80,0$ & 46.4 & 46.2 & - & 41.2 & 38.4 & 42.8 & 48.7 & 60.3 & 61.7 \\
\hline Albumin (mg/dl) & $35.0-48.0$ & - & 22 & 19 & 21 & 21 & 25.8 & 29.9 & 39.3 & 40 \\
\hline Total Cholesterol (mg/dL) & $<200$ & - & - & 431 & - & - & 338 & 215 & 180 & 147 \\
\hline Triglyceride (mg/dL) & $<150$ & - & - & 571 & - & - & 717 & 367 & 332 & 393 \\
\hline C3 & $90.0-180.0$ & - & - & - & 142 & 120 & - & 104 & 142 & - \\
\hline \multicolumn{11}{|c|}{ Urine } \\
\hline Protein & negative & negative & $4+$ & $1000 \mathrm{mg} / \mathrm{dL}$ & $400 \mathrm{mg} / \mathrm{dL}$ & $400 \mathrm{mg} / \mathrm{dL}$ & $1000 \mathrm{mg} / \mathrm{dL}$ & - & $100 \mathrm{mg} / \mathrm{dL}$ & \\
\hline Red cells & negative & negative & $3+$ & $114.3 / \mu \mathrm{l}$ & $45.47 / \mu \mathrm{l}$ & $291.41 / \mu \mathrm{l}$ & $45.47 / \mu \mathrm{l}$ & - & $45.47 / \mu \mathrm{l}$ & $6.11 / \mu \mathrm{l}$ \\
\hline Leukocytes & $0-2$ & negative & 0 & $12.3 / \mu l$ & $4.2 / \mu l$ & $4.01 / \mu \mathrm{l}$ & $0 / \mu \mathrm{l}$ & - & $25.7 / \mu l$ & $3.3 / \mu l$ \\
\hline $\begin{array}{l}\text { Ratio of total protein (in } \\
\mathrm{mg} \text { ) to creatinine (in } \mathrm{mg} \text { ) }\end{array}$ & $<0.2$ & negative & - & 23.3 & 13 & 17.3 & 10.1 & 3.6 & 0.68 & 0.4 \\
\hline
\end{tabular}

higher pain intensity (numeric pain scale 7/10) and highly debilitating, raising the suspicion of post infectious arthritis. The patient responded only after the reintroduction of low dose prednisone and hydroxychloroquine.

\section{DISCUSSION}

This case highlights the often misdiagnosed IgA dominant GN associated to staphylococcal infection that mimics IgAN mainly in cases where infection is either overlooked or not apparent. In this way, IgA dominant $\mathrm{GN}$ associated to staphylococcus infection represents a challenge in our clinical practice.

Our patient's clinical, laboratorial and histological presentation follows literature findings. In some of the major studies ${ }^{6-8}$ the diagnosis is made if at least two of the following criteria is satisfied in a patient with clinical or laboratory evidence of staphylococcal infection and onset of glomerulonephritis usually with endocapillary proliferation and exudative glomerulonephritis on LM (observed in our patient), C3 dominant/co-dominant glomerular staining or IgA-dominant or co-dominant disease together with intense C3 staining on IF microscopy (observed in our patient) and hump-shaped subepithelial deposits on EM, paired with hypocomplementemia (primarily C3).

Multiple features support IgA dominant GN over IgAN namely intercurrent culture documented staphylococcal infection, older age, history of diabetes mellitus and acute renal failure. The majority of patients present with AKI with concomitant microscopic hematuria in $83 \%$ and nephrotic range in $50 \%$ of cases. ${ }^{1}$ As in this case, infection was not apparent at the time of onset of the glomerulonephritis, as is seen in $25 \%$ of the cases described in literature. ${ }^{1}$ Although staphylococcal infection can be consistent with renal disease, glomerulonephritis can occur within a ten week period, normally between one to sixteen weeks. ${ }^{1,11,12}$ Pathological features such as endocapillary proliferation on LM and IgA co dominant deposition in IF also sustain this hypothesis. Endocapillary proliferative and exudative $\mathrm{GN}$ are reported in $63 \%$ of the cases. ${ }^{5} \mathrm{IF}$ analysis shows a dominant granular IgA glomerular staining with or without a staining for IgG and/or IgM. Staining for C3 is also seen in the majority of cases (normally stronger than IgA). EM was not performed in our patient; nevertheless a low frequency of subepithelial humps compared with poststreptococcal glomerulonephritis has been described particularly in cases in which infection is subtle or not clinically present as in this case. ${ }^{13}$ Although hypocomplementemia may be an important clue for diagnosis and is present in the majority of cases, complement levels were not consistently depressed in all case reports. ${ }^{2,14-15}$

It is extremely unlikely in this case that IgA dominant GN represents an exacerbation of a pre-existing primary IgAN as most patients are older, have no prior history of glomerulonephritis or proliferative histological findings. ${ }^{5}$

Henoch-Schonlein purpura nephritis (HSPN) associated to staphylococcus infection has also been reported in literature, making 
differential diagnosis with IgA dominant GN associated to staphylococcus infection a challenge in clinical practice. ${ }^{16,17}$ Their histopathologies are similar, presenting with proliferative glomerulonephritis and there is also renal involvement characterized by hematuria and proteinuria and in addition to being a distinct classical diagnosis in childhood, adult occurrence has also been reported. ${ }^{18}$ The diagnosis of PSH is criteria-based, supported by The European League Against Rheumatism (EULAR), the Paediatric, Rheumatology International Trials Organization (PRINTO) and Paediatric Rheumatology European Society (PRES). ${ }^{19,20}$ One study has reviewed these criteria and found applicability to adults with a diagnostic sensitivity of $99.2 \%$ and a specificity of $86 \%{ }^{21}$ Applying this diagnostic criteria helps us distinguish two identical histopathological entities, excluding HSPN due to the lack of such mandatory criteria as purpura or petechiae with lower limb predominance. Although our patient presented with proliferative glomerulonephritis with IgA deposits and has renal involvement such as proteinuria and hematuria he did not meet other minor criteria such as the diffuse acute abdominal pain or other gastrointestinal manifestations and did not present with the typical transient oligoarticular arthritis of large joints. Per definition, dominant IgA deposits are observed in the mesangium of all glomeruli; however in HSPN as a vascular disease, capillary wall staining is more frequently found and may predominate on mesangial $\lg A^{22}$, which was not verified in our patient.

Treatment is still not consistent in the literature. The standard treatment consists of completely eradicating the staphylococcal infection with an appropriate course of wisely selected antibiotics. Unfortunately, it is known that steroid therapy in staphylococcus related glomerulonephritis can precipitate severe staphylococcal sepsis and even death. Given the highly inflammatory histopathological pattern, rapid progression of renal disease and no active infection status, we opted for a steroids trial with what we considered reasonably good results. High dose steroids are generally reserved for aggressive forms of IgA nephritis and may be used for a postinfectious phase in which there is a clearly significant inflammatory pattern. ${ }^{15}$ The poor prognosis and lower recovery rate associated with IgAdominantGN is attributed to the associated comorbidities. Prognosis is guarded with less than a fifth of patients fully recovering renal function. ${ }^{5}$ The prognosis of IgAdominantGN is less favorable than typical post infectious glomerulonephritis. ${ }^{6,14}$ Hypertension, diabetes mellitus and fibrosis on kidney biopsy seem related to poor prognosis and deterioration of renal function. ${ }^{1,4}$

In addition, this current case brings to the discussion the concept of an immune mediated response resulting from slow allocation bacteria that led to different organ lesions in different temporal settings. This may also indicate a defective first-line defense (probably due to a state of chronic inflammation) in the patient's developing arthritis and glomerulonephritis that allowed the bacterial antigens to reach the synovium and glomeruli respectively. Finally, this case alerts clinicians to consider the diagnosis of IgAdominantGN when renal injury occurs and there is a previous isolation of MSSA, especially when immunosuppression states, such as diabetes mellitus, are present. Prompt treatment is warranted and may lead to full progressive recovery of renal function.

Disclosure of potential conflicts of interest: none declared.

\section{References}

1. Wehbe E, Salem C, Simon JF, Navaneethan SD, Pohl M. IgA-dominant Staphylococcus infection-associated glomerulonephritis: case reports and review of the literature. NDT Plus 2011;4:1815 .

2. Koyama A, Kobasyashi M, Yamaguchi N et al. Glomerulonephritis associated with MRSA infection: a possible role of bacterial superantigen. Kidney Int 1995;47:207.

3. Yoh K, Kobayashi M, Hirayama A, et al. A case of superantigen-related glomerulonephritis after methicillin-resistant Staphylococcus aureus (MRSA) infection. Clin Nephrol. 1997;48:311-6.

4. Koyama A, Sharmin S, Sakurai $\mathrm{H}$, et al. Staphylococcus aureus envelope antigen is a new candidate for the induction of IgA nephropathy. Kidney Int 2004;66:121-32.

5. Samih H. Nasr, VD. DÁgati. IgA-Dominant Postinfectious Glomerulonephritis: a new twist on and old disease. Nephron Clin Pract 2011;119:c18-c26.

6. Nasr SH, Fidler M, Valeri AM et al. Postinfectious Glomerulonephritis in the Eldery. J Am Soc Nephrol 2011;22:187-95.

7. Nasr SH, Radhakrishnan J, D’Agati VD. Bacterial infection-related glomerulonephritis in adults. Kidney Int 2013;83:792.

8. Nasr SH, Markowitz GS, Stokes MB, Said SM, Valeri AM, D'Agati VD. Acute postinfectious glomerulonephritis in the modern era: experience with 86 adults and review of the literature. Medicine (Baltimore) 2008;87(1):21-32.

9. Colmegna I, Cuchacovich R, Espinoza RL. HLA-B27 Associated Reactive Arthritis: Pathogenetic and clinical considerations. Clin Microbiol Rev,2004; 17(2): 348-369.

10. Khwaja A. KDIGO clinical practice guidelines for acute kidney injury. Nephron Clin Practice. 2012;120(4):c179-184

11. Glassock JR, Alvarado A, Prosek J, et al. Staphylococcus-related Glomerulonephritis and Poststreptococcal Glomerulonephritis: Why defining "Post" is important in understanding and treating infection-related glomerulonephritis. Am J Kidney Dis. 2015;65(6):826:832.

12. Kai H, Shimizu Y, Yoh K, et al. Post-MRSA infection glomerulonephritis with marked Staphylococcus aureus cell envelope antigen deposition in glomeruli. J Nephrol 2006;19:215-9.

13. Anjali A. Satoskar, Sarah Suleiman, Isabelle Ayoub et al. Staphylococcus Infection-Associated GN -Spectrum of IgA Staining and Prevalence of ANCA in a Single-Center Cohort. Clin J Am Soc Nephrol 2017;12:39-49.

14. Aliyeva N, Demir E, Caliskan Y, Yazici H, Yildiz A. A Rare Clinical Entity: Staphylococcus-Related Glomerulonephritis. Experimental and Clinical Transplantation 2017.

15. Okuyama S, Wakui H, Maki N, et al. Successful treatment of post MRSA infection glomerulonephritis with steroid therapy. Clin Nephrology 2008;70:344-7.

16. Mandai X, Aoyagi M, Nagahama K, et al. Post-staphylococcal infection Henoch-Schönlein purpura nephritis: a case report and review of the literature. Ren Fail. 2013;35(6):869-74.

17. Satoskar A, Molenda M, Scipio P, et al. Henoch-Schönlein purpura-lie presentation in IgA dominant Staphylococcus infection- associated glomerulonephritis- a diagnostic pitfall. Clin Nephrol 2013;79 (4):302-12.

18. Hočevar A, Rotar Z, Jurčićet $V$ et al. IgA vasculitis in adults: the performance of the EULAR/PRINTO/ PRES classification criteria in adults. Arthritis ResTher 2016;8:58.

19. Ozen S, Ruperto N, Dilon MJ, et al. EULAR/PRES endorsed consensus criteria for the classification of childhood vasculitis. Ann Rheum Dis 2006;65:936.

20. Ozen S, Pistorio A, lusan SM, et al. EULAR/PRINTO/PRES criteria for Henoch-Schonlein purpura, childhood polyarteritis nodosa, childhood Wegener granulomatosis and childhood Takayasu arteritis: Ankara 2008. Part II: Final classification criteria. Ann Rheum Dis 2010; 69:798.

21. Jean-Claude Davi, Ineke J. Ten Berge and Jan J. Weening. What is the difference between IgA nephropathy and Henoch-Schönlein in purpura nephritis?. Kidney International 2001;59:823-34.

22. Liv Eline Hetland, Kjaersti Susrud, Kim Lindahl and Anette Bygum. Henoch- Schönlein Purpura: A Literature Review. Acta Derm Venereol 2017;97:1160-6.

\section{Correspondence to:}

Maria Nicole Nunes Pestana

Nephrology Department, Hospital Central do Funchal

Avenida Luís de Camões, nำ57, 9000-514

E-mail: nicole.pest@gmail.com 